Research in Astron. Astrophys. 2009 Vol. 9 No. XX, 000-000

http://www.raa-journal.org http://www.iop.org/journals/raa

$\boldsymbol{R}$ esearch in

Astronomy and

Astrophysics

\title{
The cooling time of white dwarfs produced from type Ia supernovae
}

\author{
Xiang-Cun Meng ${ }^{1}$, Wu-Ming Yang ${ }^{1}$ and Zhong-Mu Li ${ }^{2,3}$ \\ ${ }^{1}$ School of Physics and Chemistry, Henan Polytechnic University, Jiaozuo, 454000, China; \\ xiangcunmeng@hotmail.com \\ 2 College of Physics and Electronic Information, Dali University, Dali, 671003, China \\ 3 National Astronomical Observatories, Chinese Academy of Sciences, Beijing, 100012, China
}

\begin{abstract}
Type Ia supernovae (SNe Ia) play a key role in measuring cosmological parameters, in which the Phillips relation is adopted. However, the origin of the relation is still unclear. Several parameters are suggested, e.g. the relative content of carbon to oxygen $(\mathrm{C} / \mathrm{O})$ and the central density of the white dwarf (WD) at ignition. These parameters are mainly determined by the WD's initial mass and its cooling time, respectively. Using the progenitor model developed by Meng \& Yang, we present the distributions of the initial WD mass and the cooling time. We do not find any correlation between these parameters. However, we notice that the range of the WD's mass decreases, while its average value increases with the cooling time. These results could provide a constraint when simulating the SN Ia explosion, i.e. the WDs with a high $\mathrm{C} / \mathrm{O}$ ratio usually have a lower central density at ignition, while those having the highest central density at ignition generally have a lower $\mathrm{C} / \mathrm{O}$ ratio. The cooling time is mainly determined by the evolutionary age of secondaries, and the scatter of the cooling time decreases with the evolutionary age. Our results may indicate that WDs with a long cooling time have more uniform properties than those with a short cooling time, which may be helpful to explain why SNe Ia in elliptical galaxies have a more uniform maximum luminosity than those in spiral galaxies.
\end{abstract}

Key words: stars: white dwarfs - stars: supernovae: general

\section{INTRODUCTION}

As one of the most widely used distance indicators, type Ia supernovae (SNe Ia) show their importance in determining cosmological parameters, which resulted in the discovery of the accelerating expansion of the universe (Riess et al. 1998; Perlmutter et al. 1999). The result was exciting and suggested the presence of dark energy. At present, SNe Ia are proposed to be cosmological probes for testing the evolution of the dark energy equation of state with time and testing the evolutionary history of the universe (Riess et al. 2007; Kuznetsova et al. 2008; Howell et al. 2009a). They were even chosen to check the consistency of general relativity (Zhao et al. 2010). When SNe Ia are applied as a distance indicator, the Phillips relation is adopted, which is a linear relation between the absolute magnitude of SNe Ia at maximum light and the magnitude drop of the B light curve during the first 15 days following the maximum (Phillips 1993). This relation implies that the brightness of SNe Ia is mainly determined by one parameter. It is generally agreed that the amount of ${ }^{56} \mathrm{Ni}$ formed during the supernova explosion dominates the maximum luminosity of SNe Ia (Arnett 1982), but the origin of the different amount of ${ }^{56} \mathrm{Ni}$ for different SNe Ia is still unclear (Podsiadlowski et al. 2008). Some numerical and synthetical results showed that metallicity has an effect on the final amount of ${ }^{56} \mathrm{Ni}$, and thus the maximum luminosity (Timmes et al. 
2003; Travaglio et al. 2005; Podsiadlowski et al. 2006; Bravo et al. 2010) and there do be some observational evidence of the correlation between the properties of SNe Ia and metallicity (Branch \& Bergh 1993; Hamuy et al. 1996; Wang et al. 1997; Cappellaro et al. 1997; Shanks et al. 2002). However, the metallicity seems not to have the ability to interpret the scatter of the maximum luminosity of SNe Ia (Timmes et al. 2003; Gallagher et al. 2008; Howell et al. 2009b). Nomoto et al. (1999, 2003) suggested that the ratio of carbon to oxygen $(\mathrm{C} / \mathrm{O})$ of a white dwarf at the moment of explosion is the dominant parameter for the Phillips relation. The higher the $\mathrm{C} / \mathrm{O}$, the larger the amount of nickel-56, and then the higher the maximum luminosity of SNe Ia. The $\mathrm{C} / \mathrm{O}$ ratio is a function of the initial mass of the WD, which then is related to the progenitor system of the SNe Ia. By comparing theory and observations, the results of Meng et al. (2009) and Meng \& Yang (2010a) upheld this suggestion. Lesaffre et al. (2006) carried out a systematic study of the sensitivity of ignition conditions for H-rich Chandra single degenerate exploders on various properties of the progenitors, and suggested that the central density of the WD at ignition may be the origin of the Phillips relation (see also Podsiadlowski et al. 2008). These authors noticed that the more massive and/or the cooler the CO WD is when accretion begins, the higher the central density is at ignition. The central density is then also related to the progenitor system. When one simulates the explosion of $\mathrm{SNe} \mathrm{Ia}$, the $\mathrm{C} / \mathrm{O}$ and the central density are always set to be free parameters (Röpke et al.2006a). It is thus interesting to analyze whether there is a correlation between the $\mathrm{C} / \mathrm{O}$ and the central density. In addition, analyzing how the initial mass of the CO WD and its cooling time vary with the delay time is also an interesting task (Greggio 2010). The purpose of this paper is to check these interesting problems.

In section 2, we describe our model. We show the results in section 3 and give discussions and conclusions in sections 4 .

\section{MODEL AND PHYSICS INPUTS}

As suggested both Nomoto et al. (1999, 2003) and Lesaffre et al. (2006), the progenitor model is the so-called single degenerate model (SD), i.e. the companion is probably a main sequence or a slightly evolved star (WD+MS) or a red-giant (WD+RG) or a helium star (WD + He star) (Whelan \& Iben 1973; Nomoto, Thielemann \& Yokoi 1984). The SD model is widely accepted and studied by many authors (Yungelson et al. 1995; Li \& van den Heuvel 1997; Hachisu et al. 1999a b; Nomoto et al. 1999, 2003; Langer et al. 2000; Han \& Podsiadlowski 2004, 2006; Chen \& Li 2007, 2009; Han 2008; Lü et al. 2009; Wang et al. 2009a b; Wang, Li \& Han 2010; Meng \& Yang 2010b.c). In this paper, we also explore the SD model. Meng \& Yang (2010a) developed a comprehensive progenitor model for SNe Ia. In the model, the mass-stripping effect by optically thick wind (Hachisu et al. 1996) and the effect of a thermally unstable disk were included (Hachisu et al. 2008; Xu \& Li 2009). The prescription of Hachisu et al. (1999a) for WDs accreting hydrogen-rich material from their companions was applied to calculate the WD mass growth. In Meng \& Yang (2010a), both the WD + MS and WD + RG scenarios are considered, i.e. Roche lobe overflow (RLOF) begins at either the MS or the RG stage. After the RLOF, the WD accretes hydrogen-rich material from its donor and increases its mass smoothly. When the mass of the WD reaches $1.378 M_{\odot}$ (Nomoto, Thielemann \& Yokoi 1984), the WD is assumed to explode as an SN Ia. They considered more than 1600 different WD close binary evolution and obtained a parameter space for SNe Ia which is summarized in an orbital period - secondary mass plane $\left(\log P^{\mathrm{i}}, M_{2}^{\mathrm{i}}\right)$. Our exploration is based on the model of Meng \& Yang (2010a).

The $\mathrm{C} / \mathrm{O}$ is a function of the initial WD mass of the progenitor system, i.e. a higher WD mass leads to a lower $\mathrm{C} / \mathrm{O}$. We then use the initial WD mass to represent the $\mathrm{C} / \mathrm{O}$. The central density is mainly determined by the initial WD mass and its cooling time, i.e. the more massive the WD and /or the longer the cooling time, the higher the central density at ignition. The cooling time of the CO WD is the time which elapses between its formation and the start of the accretion phase. We thus use the initial WD mass and its cooling time to represent the central density of a CO WD. The following is how we obtain the initial WD mass and the cooling time.

To obtain the distributions of the initial WD mass and its cooling time, we carried out a series of Monte Carlo simulations via Hurley's rapid binary evolution code (Hurley et al. 2000, 2002). The model 
grids obtained by Meng \& Yang (2010a) were incorporated into the code. We followed the evolution of $10^{7}$ binaries and a circular orbit is assumed for all binaries. The metallicity is set to solar metallicity, i.e. $Z=0.02$. The basic parameters for the simulations are as follows: (1) a single star burst; (2) the initial mass function (IMF) of Miller \& Scalo (1979); (3) the mass-ratio distribution is constant; (4) the distribution of separations is constant in $\log a$ for wide binaries, where $a$ is the orbital separation; (5) the common envelope (CE) ejection efficiency $\alpha_{\mathrm{CE}}$, which denotes the fraction of the released orbital energy used to eject the CE, is set to be either 1.0 or 3.0 (see Meng \& Yang (2010a) for details of the parameter input).

There are three channels that produce WD + MS systems and one channel that produce a WD + RG system according to the situation of the primary in a primordial system at the onset of the first RLOF, i.e. the He star channel, the EAGB channel and the TPAGB channel (see Meng \& Yang 2010a for details about the channels). The formed SD systems continue to evolve and the secondaries may fill their Roche lobes at a later stage, leading to the start of the RLOF. We assume that if the initial orbital period, $P_{\mathrm{orb}}^{\mathrm{i}}$, and the initial secondary mass, $M_{2}^{\mathrm{i}}$, of an SD system is located in the appropriate regions in the $\left(\log P^{\mathrm{i}}, M_{2}^{\mathrm{i}}\right)$ plane for SNe Ia at the onset of RLOF, an SN Ia is then produced. The cooling time is then the difference between the formation time of an SD system and the starting time of the RLOF.

\section{RESULTS}

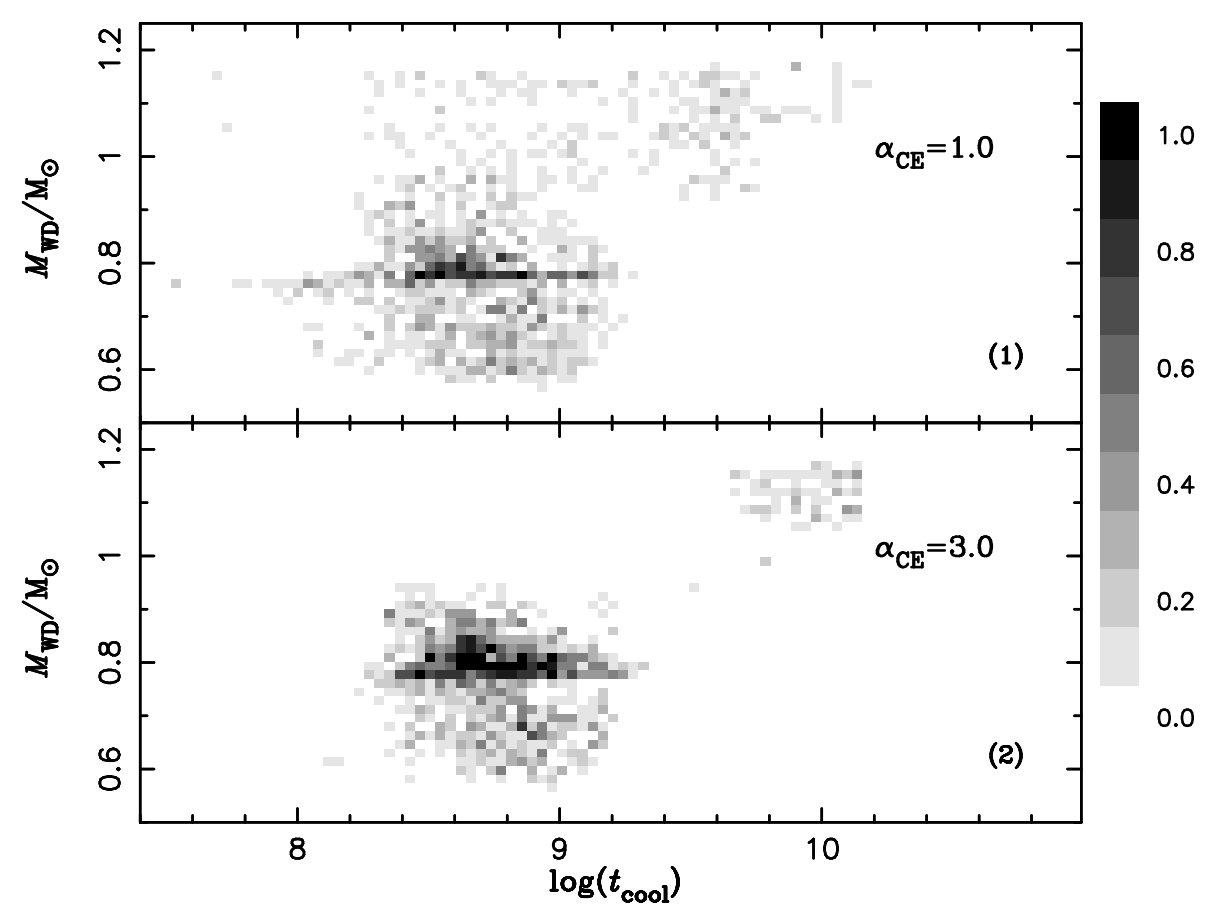

Fig. 1 Distributions of initial WD masses and their cooling times for different $\alpha_{\mathrm{CE}}$.

\subsection{Distributions of the initial WD mass and their cooling time}

In Fig. 11 we show the distributions of the initial WD mass and its cooling times for different $\alpha_{\mathrm{CE}}$. We see from the figure that $\alpha_{\mathrm{CE}}$ does not significantly affect the distributions. We do not find any correlation between the initial WD mass and its cooling time, which means that taking the $\mathrm{C} / \mathrm{O}$ ratio and the central 


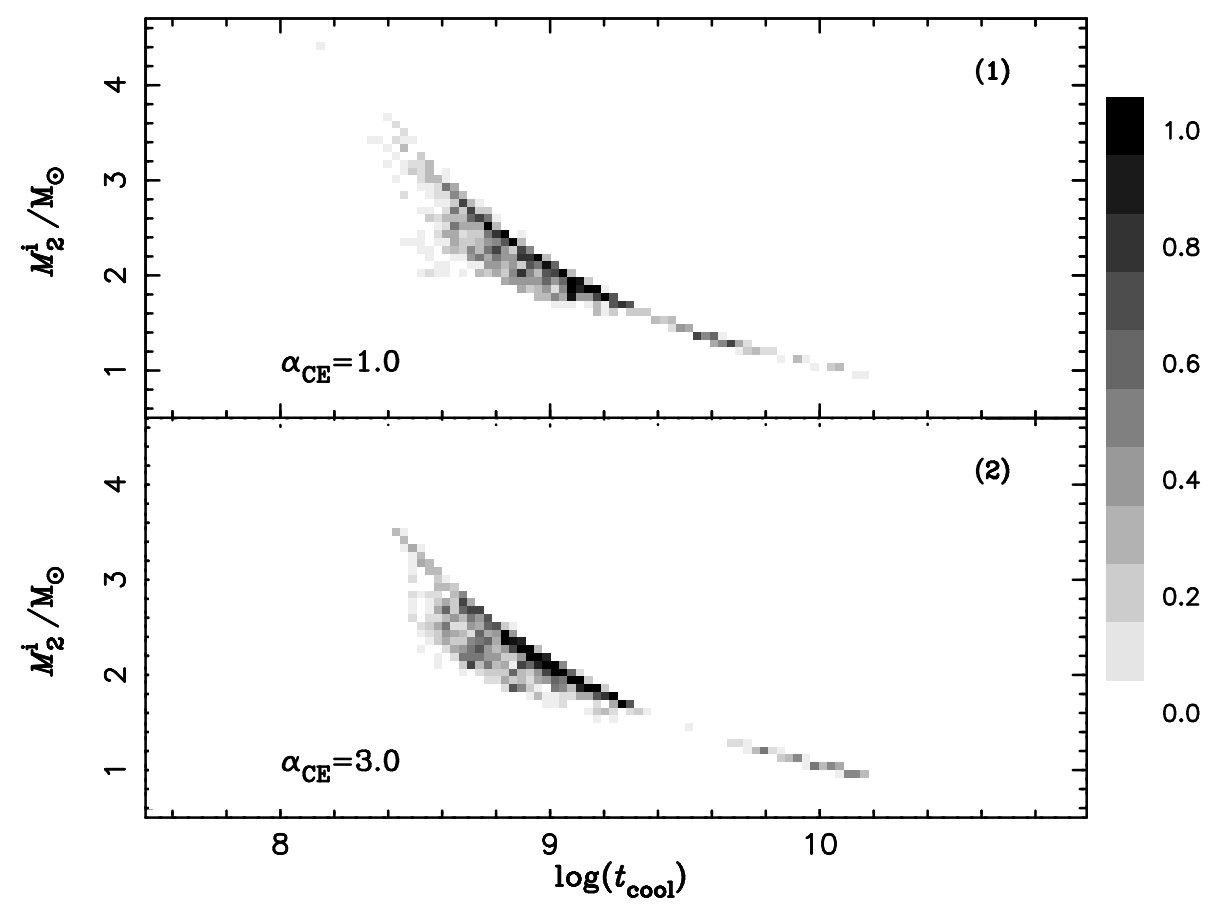

Fig. 2 Distributions of the initial secondary mass and the cooling times of CO WDs for different $\alpha_{\mathrm{CE}}$.

density of a CO WD as free parameters is reasonable. However, the range of the WD mass decreases and the mean value of the WD mass increases with the cooling time, i.e. the range of the cooling time increases with the WD mass (see Fig. 9 in Meng \& Yang 2010a). In addition, it is important to notice that there are some events with a very long cooling time, i.e. several Gyrs, although the majority of CO WDs have a cooling time shorter than 1 Gyr. The models in Lesaffre et al. (2006) include cooling time ages of $\simeq 1 \mathrm{Gyr}$ at most. For a long cooling time, the CO WD may become more degenerate before the start of the accretion phase, and some other process like $\mathrm{C}$ and $\mathrm{O}$ separation or crystallization may occur, and dominate the properties of the CO WD (Fontaine et al. 2001). The simulation of the SNe Ia explosion under extremely degenerate conditions should then be a more important problem than that presented in literatures.

We also investigate the distributions of the initial orbital period and the cooling time of a CO WD. Similar to the distribution of the initial WD mass and its cooling time, no correlation between the initial orbital period and the cooling time of a CO WD was discovered.

\subsection{Distributions of the initial secondary mass and the cooling time}

We present the distributions of the initial secondary mass and the cooling time for different $\alpha_{\mathrm{CE}}$ values in Fig. 2. Although $\alpha_{\mathrm{CE}}$ also does not significantly affect the distributions, these distributions differ remarkably from those of the initial WD mass and the cooling time. The cooling time is highly relevant to the secondary mass. It is clearly shown in Fig. 2 that there is an upper limit of the cooling time for a given secondary mass, and most of the events have a cooling time equal to the upper limit. The upper limit is derived from a constraint that the cooling time should be smaller than the evolutionary age of the secondary. However, there is still a scatter in the cooling time for a certain secondary mass, which originated from the different initial orbital period for an initial system with given WD and secondary 


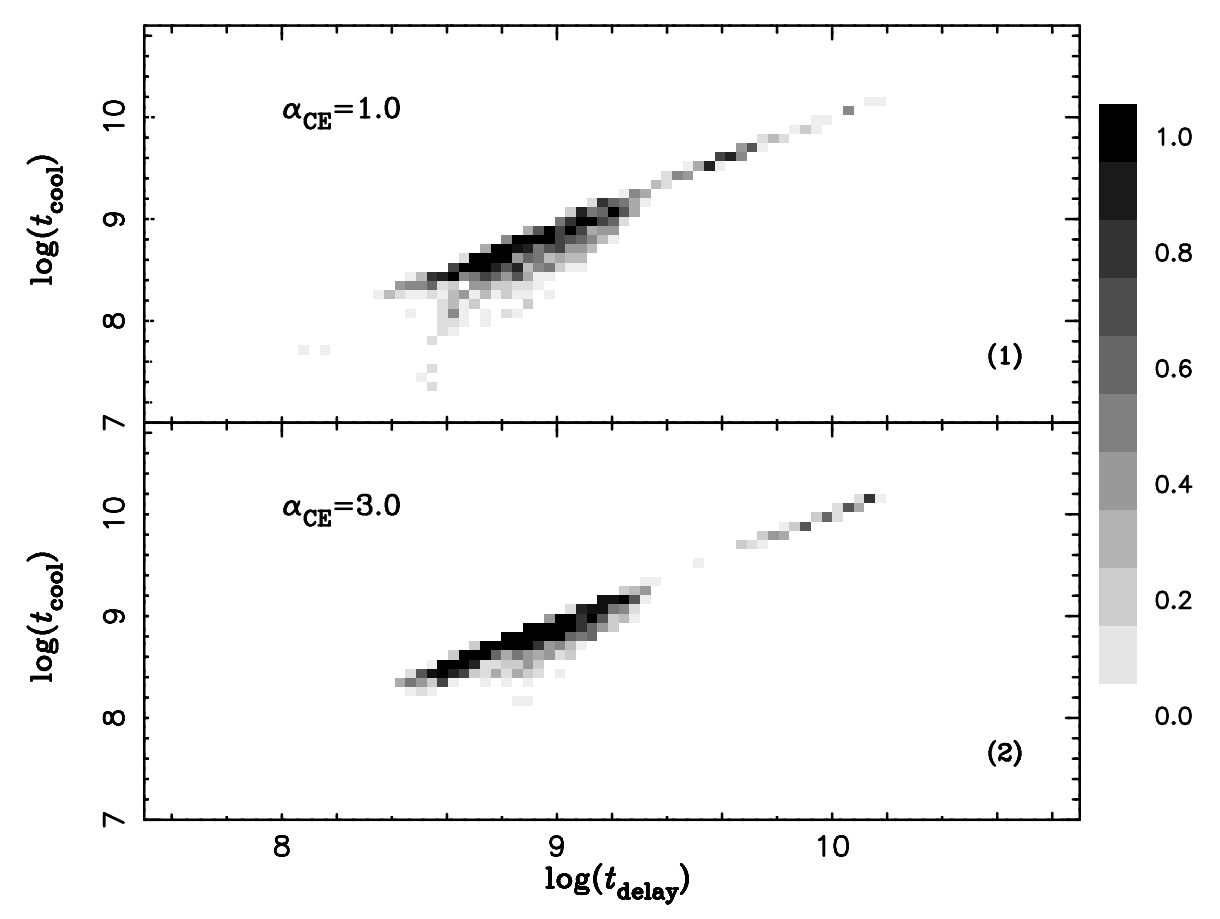

Fig. 3 Relation between the cooling time of CO WDs and the delay time of SNe Ia for different $\alpha_{\mathrm{CE}}$.

masses. The shorter the initial orbital period, the earlier the onset of the RLOF between the WD and secondary, and then the shorter the cooling time. In addition, the scatter decreases with the decrease of secondary mass, and when the cooling time is larger than $2 \mathrm{Gyr}$, the scatter almost disappears.

\subsection{Relation between the cooling time of CO WDs and the delay time of SNe Ia}

It is interesting to analyze how the WD cooling time varies with the delay time of SNe Ia. In Fig. 3 the relation between the cooling time and the delay time is presented. The relation is very tight, and there is also an upper limit of the cooling time for a given delay time. This result is directly from the relation between the secondary mass and delay time. For most of the progenitor systems with a certain secondary, the delay time is the evolutionary lifetime of the secondary (Meng \& Yang 2010a; Greggio 2010). So, using the secondary evolutionary time as the delay time of SNe Ia is an excellent approximation when one applies an analytic method to study the delay time of SNe Ia Greggio 2010). There is also a scatter for the relation between the cooling time and the delay time, and the scatter decreases with the delay time. When the delay time is larger than $2 \mathrm{Gyr}$, the cooling time is almost equal to the delay time.

\section{DISCUSSION AND CONCLUSIONS}

In this paper, we do not find a correlation between the initial WD mass and its cooling time. Since the $\mathrm{C} / \mathrm{O}$ ratio is a function of the initial WD mass and the central density for a WD with given initial mass is mainly determined by the cooling time (Nomoto et al. 1999, 2003; Lesaffre et al. 2006), our results may imply that the $\mathrm{C} / \mathrm{O}$ ratio and the central density at ignition are free parameters when simulating an $\mathrm{SNe}$ Ia explosion. However, our results still provide a constraint when simulating these types of explosions, i.e. the cooling time of WDs with an initial mass less than $1 M_{\odot}$ is generally shorter than 1 Gyr, but it may be as long as 15 Gyr for WDs with an initial mass larger than $1 M_{\odot}$ (see Fig. 1). Because a 
high initial WD mass means a lower $\mathrm{C} / \mathrm{O}$, and a massive WD and a long cooling time leads to a high central density at ignition (Nomoto et al. 1999, 2003; Lesaffre et al. 2006), the above result could imply that WDs with a high $\mathrm{C} / \mathrm{O}$ usually have a lower central density at ignition, but those having the highest central density at ignition generally have a lower $\mathrm{C} / \mathrm{O}$.

We also checked the effect of metallicity on the distributions of the initial WD mass and the cooling time by $Z=0.001$ (Meng, Li \& Yang 2010) and no significant effect was found. Theory and observations did confirm that the effect of metallicity cannot explain the scatter in the maximum luminosity of SNe Ia (Timmes et al. 2003; Gallagher et al. 2008; Howell et al. 2009b). Metallicity should, at most, be the secondary parameter for the Phillips relation, i.e. it is the origin for the scatter of the Phillips relation (Podsiadlowski et al. 2006, 2008). Furthermore, even regarding the secondary parameter, there is not a consensus. Mazzali \& Podsiadlowski (2006) suggested that a variation of the relative content of $\left({ }^{54} \mathrm{Fe}+{ }^{58} \mathrm{Ni}\right)$ versus ${ }^{56} \mathrm{Ni}$ may be responsible for the observed scatter of the Phillips relation. However, Kasen, Röpke \& Woosley (2009) argued that the breaking of spherical symmetry is a critical factor in determining both the Phillips relation and the observed scatter around it. Then, the origin of both the Phillips relation and the scatter of the relation still should be investigated carefully.

We found that the range of the initial WD mass decreases and the average WD mass increases with the cooling time, which is similar to the relation between the initial WD mass and the delay time found by Meng \& Yang (2010a). In addition, the scatter of the cooling time also decreases with delay time. These results may indicate that the difference among the WDs with s short cooling time could be huge, but the properties of WDs with a long cooling time might be more uniform (see from Fig. 1 that all the WDs with a cooling time longer than several Gyr have a mass larger than $\left.1.0 M_{\odot}\right)$. Since a long cooling time is equivalent to a long delay time (see Fig. 3), our results may imply that the properties of SNe Ia with long delay times might be more uniform than those with short delay times. It is widely known that there exists a scatter of the maximum luminosity of SNe Ia, and the scatter is affected by its environment. The most luminous SNe Ia always occur in spiral galaxies, but both spiral and elliptical galaxies are hosts for dimmer SNe Ia, which lead to a dimmer mean peak brightness in elliptical than in spiral galaxies, i.e. the maximum luminosity of SNe Ia hosted in elliptical galaxies is more uniform (Hamuy et al. 1996). These properties may be qualitatively interpreted by the model developed by Meng \& Yang (2010a), at least if the $\mathrm{C} / \mathrm{O}$ ratio is the origin of the Phillips relation. However, the quantitative study by Röpke et al. (2006a) showed that the $\mathrm{C} / \mathrm{O}$ has only little impact on the amount of produced ${ }^{56} \mathrm{Ni}$. It should be noticed that the results in Röpke et al. (2006a) are model dependent, and are much different from those of Nomoto et al. (1999, 2003). Considering that the central density is mainly determined by the initial mass of the WD and its cooling time, the results in Figs. 1 and 3, i.e. the average values of the WD mass and the cooling time increase with the delay time, which means that the average of the central density increases with the delay times of SNe Ia. A high central density at ignition leads to a larger amount of the produced ${ }^{56} \mathrm{Ni}$ (Röpke et al. 2006a). The effect of the central density on the amount of ${ }^{56} \mathrm{Ni}$ seems then to be opposite with observations of Hamuy et al. (1996). Furthermore, the variation of the amount of ${ }^{56} \mathrm{Ni}$ derived from the central density only amounts to about $7 \%$, which can not be used to interpret the variation in the maximum luminosity of SNe Ia (Röpke et al. 2006a). Perhaps, the C/O ratio, the central density and the metallicity all contribute to the variation of the maximum luminosity of SNe Ia (Röpke et al. 2006b). Then, which is the dominant parameter for the Phillips relation is still an open queation. In addition, as the cooling/delay time increases, the CO WDs become more and more degenerate, and even crystallization may occur (Fontaine et al. 2001). What is the effect of the crystallization on the amount of ${ }^{56} \mathrm{Ni}$ should be an interesting problem.

Acknowledgements This work was supported by Natural Science Foundation of China under grant no. 10963001 and the Project of the Fundamental and Frontier Research of Henan Province under grant no. 102300410223. 


\section{Appendix A: THIS SHOWS THE USE OF APPENDIX}

\section{References}

Arnett W.D., 1982, ApJ, 253, 785

Branch D., \& van den Bergh S.V., 1993, AJ, 105, 2231

Bravo E., Domínguez I., Badenes C. et al., 2010, ApJ, 711, L66

Cappellaro E., Turatto M., Tsvetkov D.Y., Bartunov O.S., Pollas C., Evans R., Hamuy M., 1997, A\&A, 322,431

Chen W., Li X., 2007, ApJ, 658, L51

Chen, W., Li X., 2009, ApJ, 702, 686

Fontaine G., Brassard P., Bergeron P., 2001, PASP, 113, 409

Gallagher, J. S., Garnavich, P. M., Caldwell, N., Kirshner, R. P., Jha, S. W., Li, W., Ganeshalingam, M., Filippenko,

A. V., 2008, ApJ, 685, 752

Greggio L., 2010, MNRAS, accepted, arXiv: 1001.3033

Hachisu I., Kato M., Nomoto K., ApJ, 1996, 470, L97

Hachisu I., Kato M., Nomoto K., Umeda H., 1999a, ApJ, 519, 314

Hachisu I., Kato M., Nomoto K., 1999b, ApJ, 522, 487

Hachisu I., Kato M., Nomoto K., 2008, ApJ, 679, 1390 (arXiv: 0710.0319)

Hamuy M., Phillips M.M., Schommer R.A., Maza J., Avilés R., 1996, AJ, 112, 2391

Han Z., Podsiadlowski Ph., 2004, MNRAS, 350, 1301

Han Z., Podsiadlowski Ph., 2006, MNRAS, 368, 1095

Han Z., 2008, ApJ, 677, L109

Howell, D.A. et al., 2009a, arXiv: 0903.1086

Howell, D.A. et al., 2009b, ApJ, 691, 661

Hurley J.R., Pols O.R., Tout C.A., 2000, MNRAS, 315, 543

Hurley J.R., Tout C.A., Pols O.R., 2002, MNRAS, 329, 897

Kasen D., Röpke F.K., Woosley S.E., 2009, Nature, 460, 869

Kuznetsova N., Barbary K., Connolly B. et al., 2008, ApJ, 673, 981

Langer N., Deutschmann A., Wellstein S. et al., 2000, A\&A, 362, 1046

Lesaffre P., Han Z., Tout C. A. et al., 2006, MNRAS, 368, 187

Li X.D., van den Heuvel E.P.J., 1997, A\&A, 322, L9

Lü, G., Zhu, C. Wang, Z., Wang, N., 2009, MNRAS, 396, 1086, arXiv:0903.2636

Mazzali P. A., Podsiadlowski P., 2006, MNRAS, 369, L19

Meng X., Chen X., Han Z., 2009, MNRAS, 395, 2103

Meng X., Yang W., 2010a, ApJ, 710, 1310

Meng X., Yang W., 2010b, MNRAS, 401, 1118

Meng X., Yang W., 2010c, A\&A, 516, A47, arXiv: 1003.2659

Meng X., Li Z., Yang W., 2010, PASJ, submitted

Miller G.E., Scalo J.M., 1979, ApJS, 41, 513

Nomoto K., Thielemann F-K., Yokoi K., 1984, ApJ, 286, 644

Nomoto K., Umeda H., Hachisu I. Kato M., Kobayashi C., Tsujimoto T., 1999, in Truran J., Niemeyer T., eds, Type Ia Suppernova :Theory and Cosmology.Cambridge Univ. Press, New York, p.63

Nomoto K., Uenishi T., Kobayashi C. Umeda H., Ohkubo T., Hachisu I., Kato M., 2003, in Hillebrandt W., Leibundgut B., eds, From Twilight to Highlight: The Physics of supernova, ESO/Springer serious "ESO Astrophysics Symposia” Berlin: Springer, p.115

Podsiadlowski P., Mazzali P.A., Lesaffre P., Wolf C., Förster F., 2006, arXiv: 0608324

Podsiadlowski P., Mazzali P., Lesaffre P., Han, Z., Förster F., 2008, NewAR, 52, 381

Perlmutter S. et al., 1999, ApJ, 517, 565

Phillips M.M., 1993, ApJ, 413, L105

Riess A. et al., 1998, AJ, 116, 1009

Riess A. et al., 2007, ApJ, 659, 98

Röpke F.K., Gieseler M., Reinecke M. et al., 2006a, A\&A, 453, 203 
Röpke F.K., Gieseler M., Hillebrandt W., 2006b, arXiv:0609459

Shanks T., Allen P.D., Hoyle F. et al., 2002, ASPC, 283, 274

Timmes F.X., Brown E.F., Truran J.W., 2003, ApJ, 590, L83

Travaglio C., Hillebrandt W., Reinecke M., 2005, A\&A, 443, 1007

Wang, B., Meng, X., Chen, X., Han, Z., 2009a, MNRAS, 395, 847

Wang, B., Chen, X., Meng, X., Han, Z., 2009b, ApJ, 701, 1540

Wang, B., Li, X., Han, Z., 2010, MNRAS, 401, 2729

Wang L., Höflich P., Wheeler J.C., 1997, ApJ, 483, L29

Whelan J., Iben I., 1973, ApJ, 186, 1007

Xu X. \& Li X., 2009, A\&A, 495, 243

Yungelson L., Livio M., Tutukou A. Kenyon S.J., 1995, ApJ, 447, 656

Zhao G., Giannantonio T., Pogosian L. et al., 2010, arXiv: 1003.0001 